\title{
Vestibular Neuritis With Minimal Canal Paresis: Characteristics and Clinical Implication
}

\author{
Hyun Ji Kim · Dae-Young Kim · Jun Ha Hwang · Kyu-Sung Kim \\ Department of Otorhinolaryngology, Inha University School of Medicine, Incheon, Korea
}

Objectives. To analyze the clinical characteristics of vestibular neuritis patients with minimal canal paresis (canal paresis $<25 \%)$.

Methods. Patients clinically diagnosed with vestibular neuritis and treated at our institute $(n=201)$ underwent otoneurological examination and vestibular function tests. Patients were categorized in terms of the results of caloric testing (canal paresis $<25 \%, n=58$; canal paresis $\geq 25 \%, n=143$ ). Clinical characteristics and laboratory outcomes were compared between two groups.

Results. Existence of underlying diseases, preceding symptoms, and direction of spontaneous nystagmus were not different between the groups. The mean duration of spontaneous nystagmus was shortest in the minimal canal paresis group $(P<0.001)$ and the direction of spontaneous nystagmus changed more frequently in this group $(P<0.001)$ during recovery. Among the subgroup with minimal canal paresis, only $29.5 \%$ had an abnormal finding on the rotatory chair test, as compared to $81.5 \%$ of the canal paresis group. The minimal canal paresis group showed higher sensory organization test scores in computerized dynamic posturography.

Conclusion. Patients with minimal canal paresis (canal paresis $<25 \%$ ) show similar clinical manifestations as conventional vestibular neuritis patients, but have faster recovery of symptoms and a higher incidence of recovery nystagmus. This finding support that the minimal canal paresis could be considered as a milder type of vestibular neuritis.

Keywords. Vestibular Neuritis; Canal Paresis; Spontaneous Nystagmus; Vertigo

\section{INTRODUCTION}

Vestibular neuritis $(\mathrm{VN})$ is characterized by rotatory vertigo with an acute onset, with associated nausea, vomiting, and generalized imbalance. In general, the dizziness lasts from several hours to several days with gradual, definite improvement throughout its course. Patients usually show horizontal torsional spontaneous nystagmus toward the healthy side, an abnormal finding on the head-impulse test toward the affected side, and a deviation

- Received July 13, 2016

Revised August 4, 2016

Accepted August 12, 2016

- Corresponding author: Kyu-Sung Kim

Department of Otorhinolaryngology, Inha University School of Medicine,

27 Inhang-ro, Jung-gu, Incheon 22332, Korea

Tel: +82-32-890-3620, Fax: +82-32-890-3580

E-mail: stedman@inha.ac.kr of the subjective visual vertical toward the affected side. $\mathrm{VN}$ is known that the third most common cause of peripheral vertigo. It accounts for $7 \%$ of the patients at outpatient clinics specializing in the treatment of vertigo [1].

A diagnosis of $\mathrm{VN}$ depends on a history of spontaneous, rotatory vertigo and physical assessments that are consistent with a unilateral peripheral vestibular loss without other cochlear symptoms. History and physical examination alone are usually adequate for diagnosis. Although VN has distinctive clinical characteristics, it used to be thought to be a diagnosis of exclusion due to its ambiguous diagnostic criteria. Relevant differential diagnoses are required for central lesions, such as pseudovestibular neuritis due to acute cerebellar infarctions or pontomedullary lesions, as well as other peripheral vestibular diseases, vestibular migraine, and monosymptomatic Meniere's disease [1-3].

Copyright @ 2017 by Korean Society of Otorhinolaryngology-Head and Neck Surgery.

This is an open-access article distributed under the terms of the Creative Commons Attribution Non-Commercial License (http://creativecommons.org/licenses/by-nc/4.0)

which permits unrestricted non-commercial use, distribution, and reproduction in any medium, provided the original work is properly cited. 
The diagnostic criteria for VN suggested by Coats in 1969 are as follows: (1) acute unilateral peripheral vestibular disease without hearing loss; that (2) commonly occurs in middle-aged adults; (3) isolated prolonged vertigo; (4) unilateral canal paresis (CP) on caloric testing; and (5) complete recovery of symptoms within 6 months [4]. Using the Coats criteria, unilateral CP became an important factor in the diagnosis of VN. However, there have been some conflicting reports about $\mathrm{CP}$ in $\mathrm{VN}[5,6]$. In 1993, Cooper [7] presented three criteria for diagnosing VN: a sudden onset of vertigo, an absence of cochlear symptoms, and an absence of associated neurological symptoms and signs. A reduced or absent response to the caloric test was considered a nonessential criterion [7]. No standardized diagnostic criteria for VN have been suggested to date, and the importance of $\mathrm{CP}$ in diagnosing $\mathrm{VN}$ is still controversial, while few studies have reported on the minimal canal paresis (MCP) in VN patients.

In this study, we retrospectively investigated clinical characteristics of VN patients with minimal CP through the comparison of conventional $\mathrm{VN}$ patients and analyzed the correlation of clinical features and the degree of $\mathrm{CP}$.

\section{MATERIALS AND METHODS}

Patients diagnosed with VN and treated between 2008 and 2015 and who had undergone an otoneurological examination and vestibular function test were included. The inclusion criteria were: (1) first attack of acute vertigo; (2) symptoms of dizziness for more than hours; (3) spontaneous nystagmus to the healthy side. The exclusion criteria were an experience previous vertigo attacks, combined hearing impairment or tinnitus, abnormal findings in the central nervous system examined by otoneurological assessment, and abnormal findings in vestibular evoked myogenic potential (VEMP) tests. Patients with pathological findings on brain imaging (magnetic resonance imaging or computed tomography) were also excluded. Patients were divided into two groups according to the results of their caloric tests into a minimal canal paresis group ( $\mathrm{CP}<25 \%$; MCP group, $\mathrm{n}=58$ ) and a conventional $\mathrm{VN}$ group ( $\mathrm{CP} \geq 25 \%$; $\mathrm{CP}$ group, $\mathrm{n}=143)$.

Bithermal caloric irrigation was performed for each ear using water $(50 \mathrm{~mL})$ with a temperature of $30^{\circ} \mathrm{C}$ and $44^{\circ} \mathrm{C}$ for cold and warm irrigation, respectively. The maximum velocity of the slow

\section{H I $G$ G H L I I G H T S}

- Patients with minimal canal paresis have similar clinical manifestations as conventional vestibular neuritis patients.

- Patients with minimal canal paresis showed more rapid resolution of symptoms.

- Not only the existence of canal paresis, but also the value of canal paresis could help to predict the clinical course. phase component was analyzed. $\mathrm{CP}$ was quantified with the Jongkees' formula [8]. A CP value $\geq 25 \%$ was considered as pathological, according to the normative data from our laboratory.

For the sinusoidal harmonic acceleration (SHA) test, patients were positioned with their heads pitched at $30^{\circ}$ forward at a rotatory chair to achieve maximum stimulation of the horizontal semicircular canals. Sinusoidal oscillation for the earth's vertical axis was carried out at frequencies of $0.01,0.02,0.04,0.08$, and $0.16 \mathrm{~Hz}$. The maximum velocity was $50 \%$ s. Test was performed in the dark and alertness was assured by conversation. Gain, symmetry and phase lead were calculated from the slow phase velocity and chair velocity.

All patients were also evaluated the sensory organization test (SOT) scores with computerized dynamic posturography (EquiTest System, Neurocom Inc., Clackamas, OR, USA). The SOT evaluates postural stability using systematic changes in somatosensory as well as visual information available to the patients. Equilibrium scores from the SOT were used for our analysis. An equilibrium score ranged between $0 \%$ (fall) and $100 \%$ (did not sway at all). The mean equilibrium score for 3 trials under each SOT condition was measured for each patient. Typically, SOT uses 6 conditions; among them conditions 4, 5, and 6 , which are related to vestibular function, were used in this study.

Continuous variables were reported using the mean and standard error, and categorical variables are described using absolute and relative frequencies. Categorical variables were estimated using chi-square tests. All statistical analyses with IBM SPSS ver. 20.0 (IBM Co., Armonk, NY, USA). P-values of 0.05 were used as the value for statistical significance.

\section{RESULTS}

The general characteristics of the $201 \mathrm{VN}$ patients treated at our institute and who participated in this study are described in Table 1 . The group of patients consisted of 116 men and 85 women, with an overall mean age of $55 \pm 10$ years (range, 23 to 77 years). There were no significant differences in epidemiological factors, such as distribution of age and sex, between the two groups. There were also no differences in the proportion of underlying diseases, such as diabetes mellitus, hypertension, or hyperlipidemia between the groups. Seven patients $(12.1 \%)$ of the MCP group had experienced upper respiratory infection before the dizziness symptom onset, as did 15 patients $(10.5 \%)$ in CP group. Only 8 patients $(13.8 \%)$ in the MCP group and 11 patients $(7.7 \%)$ in the $\mathrm{CP}$ group had an accompanying headache. There was no differences in the comparison of the two groups in the proportion of patients with a headache $(P=0.317)$. There was a significant difference in the duration of spontaneous nystagmus. In the MCP group, this lasted for $3.0 \pm 1.7$ days, as compared to $4.2 \pm 1.9$ days in the $\mathrm{CP}$ group $(P<0.001)$. Patients with $\mathrm{MCP}$ more frequently showed a change in the direction of spon- 
taneous nystagmus, which was considered recovery nystagmus, during the admission period than did the CP group $(P<0.001)$.

For caloric testing, the unilateral $\mathrm{CP}$ of the entire study group $(\mathrm{n}=201)$ was $40.8 \% \pm 7.2 \%$. The values of CP were $11.2 \% \pm$ $7.6 \%$ (MCP group) and $52.5 \% \pm 15.7 \%$ (CP group), which differed significantly $(P<0.001)$ The mean slow phase peak velocity was $24.0 \pm 9.8^{\circ} / \mathrm{sec}$ in the MCP group and $15.7 \pm 5.5^{\circ} / \mathrm{sec}$ in the $\mathrm{CP}$ group $(P<0.001)$. For the direction of directional preponderance (DP), there was a difference between two groups

Table 1. Demographic and clinical characteristics of patients with and without canal paresis $(\mathrm{CP})$

\begin{tabular}{lccc}
\hline Variable & $\mathrm{CP}<25 \%$ & $\mathrm{CP} \geq 25 \%$ & $P$-value \\
\hline Age $(\mathrm{yr})$ & $58 \pm 7$ & $52 \pm 10$ & 0.583 \\
Sex & & & \\
$\quad$ Male & $28(48.3)$ & $88(61.5)$ & 0.532 \\
$\quad$ Female & $30(51.7)$ & $55(38.5)$ & 0.464 \\
Underlying disease & & & \\
Diabetes mellitus & $9(15.5)$ & $11(7.7)$ & 0.285 \\
Hypertension & $19(32.7)$ & $44(30.7)$ & 0.381 \\
Hyperlipidemia & $6(10.3)$ & $27(18.9)$ & 0.298 \\
Preceding upper respiratory & $7(12.1)$ & $15(10.5)$ & 0.415 \\
$\quad$ infection & & & \\
Accompanying headache & $8(13.8)$ & $11(7.7)$ & 0.317 \\
Duration of SN (day) & $3.0 \pm 1.7$ & $4.2 \pm 1.9$ & $<0.001^{*}$ \\
Direction change of SN & $14(24.1)$ & $8(5.6)$ & $<0.001^{*}$ \\
\hline
\end{tabular}

Values are presented as number (\%) or mean \pm standard error.

$\mathrm{SN}$, spontaneous nystagmus.

${ }^{*} P<0.05$.

Table 2. Results of caloric testing between the groups

\begin{tabular}{lccc}
\hline Variable & CP $<25 \%$ & $\mathrm{CP} \geq 25 \%$ & $P$-value \\
\hline Canal paresis (\%) & $11.2 \pm 7.6$ & $52.5 \pm 15.7$ & $<0.001^{*}$ \\
DP $(\%)$ & $12.2 \pm 9.6$ & $26.6 \pm 12.5$ & 0.055 \\
Direction of DP & & & $<0.001^{*}$ \\
$\quad$ Ipsilateral & $24(41.4)$ & $17(11.9)$ & \\
$\quad$ Contralateral & $34(58.6)$ & $126(88.1)$ &
\end{tabular}

Values are presented as number (\%) or mean \pm standard error. $\mathrm{CP}$, canal paresis; DP, directional preponderance.

${ }^{\star} P<0.05$.

Table 3. Results of sinusoidal harmonic acceleration testing

\begin{tabular}{lrrc}
\hline Variable & $\mathrm{CP}<25 \%$ & $\mathrm{CP} \geq 25 \%$ & $P$-value \\
\hline Gain $(\mathrm{Hz})$ & & & \\
0.01 & $0.35 \pm 0.10$ & $0.21 \pm 0.12$ & $<0.001^{*}$ \\
0.02 & $0.41 \pm 0.09$ & $0.30 \pm 0.08$ & $<0.001^{*}$ \\
0.04 & $0.52 \pm 0.08$ & $0.39 \pm 0.09$ & $<0.001^{*}$ \\
0.08 & $0.50 \pm 0.09$ & $0.43 \pm 0.09$ & $0.023^{*}$ \\
0.16 & $0.58 \pm 0.28$ & $0.49 \pm 0.20$ & 0.137 \\
Phase lead & $7(12.1)$ & $85(59.4)$ & $<0.001^{*}$ \\
Asymmetry & $18(31.0)$ & $125(87.4)$ & $<0.001^{*}$ \\
\hline
\end{tabular}

Values are presented as number (\%) or mean \pm standard error. $\mathrm{CP}$, canal paresis.

${ }^{*} P<0.05$. significantly. Patients with CP showed contralateral side DP more frequently (88.1\%) than patients with normal CP $(58.6 \%$; $P<0.001$ ) (Table 2).

The results of SHA testing are described in Table 3. Phase lead was shown in 85 patients $(59.4 \%)$ in the $\mathrm{CP}$ group and 7 patients $(12.1 \%)$ in the MCP group $(P<0.001)$. Asymmetry was shown in 18 patients $(31.0 \%)$ in the MCP group versus 125 patients $(87.4 \%)$ in the $\mathrm{CP}$ group $(P<0.001)$. Vestibulo-ocular reflex (VOR) gain at $0.01,0.02,0.04,0.08$, and $0.16 \mathrm{~Hz}$ was $0.35 \pm 0.10,0.41 \pm 0.09,0.52 \pm 0.08,0.50 \pm 0.09$, and $0.58 \pm$ 0.28 , respectively, in the MCP group and $0.21 \pm 0.12,0.30 \pm$ $0.08,0.39 \pm 0.09,0.43 \pm 0.09$, and $0.49 \pm 0.20$ in the $\mathrm{CP}$ group. Except at $0.16 \mathrm{~Hz}$, the $\mathrm{CP}$ group patients showed significantly lower gain than the MCP group. The difference in the gain was larger in the lower frequencies (Fig. 1). More patients in the CP group showed asymmetry on SHA testing, as compared to the MCP group $(P<0.001)$.

The SOT results are shown in Fig. 2. The mean equilibrium scores for condition 4, condition 5 , and condition 6 were as follows: condition 4, 79.1 \pm 6.39 (MCP group) vs. $79.1 \pm 5.72$ (CP group); condition $5,63.3 \pm 9.34$ (MCP group) vs. $52.8 \pm 12.6$ (CP group); and condition $6,63.0 \pm 11.1$ (MCP group) vs. $54.0 \pm$ 11.9 (CP group). The $\mathrm{CP}$ group showed significantly lower equilibrium scores than the MCP group in both conditions 5 and 6 $(P<0.001)$ (Fig. 2).

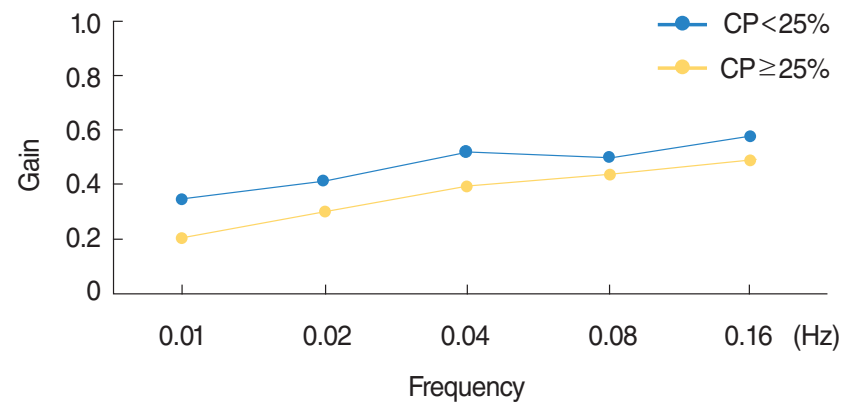

Fig. 1. Gain in sinusoidal harmonic acceleration (SHA) testing of the patients. There were significant differences between the groups in $0.01,0.02,0.04$, and $0.08 \mathrm{~Hz}$. CP, canal paresis.

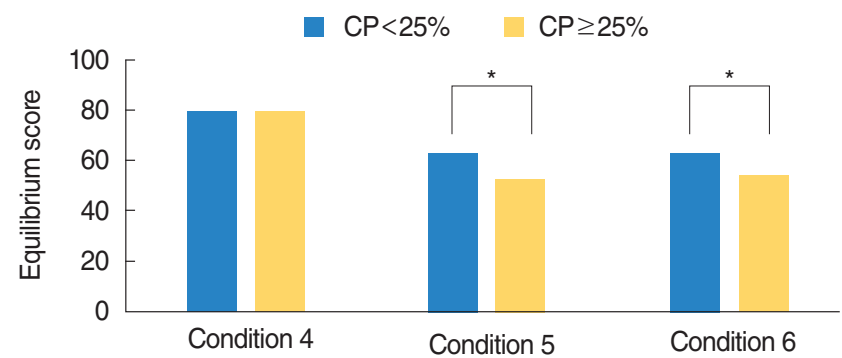

Fig. 2. Comparison of equilibrium scores of the sensory organization tests between patients with and without minimal canal paresis (CP). There were statistically significant differences both in condition 5 and condition 6. ${ }^{\star} P<0.05$ 


\section{DISCUSSION}

$\mathrm{VN}$ is a common cause of peripheral vertigo [1]. There are no conclusive diagnostic tests for VN. Molecular biological studies have revealed that $\mathrm{VN}$ could be caused by a latent reactivation of herpes simplex virus type 1 infection in the vestibular ganglia $[9,10]$. However, it is difficult to detect the virus in every clinical case, which makes it difficult to use viral infection as a diagnostic marker for VN. For objective documentation of VN, a vestibular function test, such as a caloric test, rotatory chair test, or video head-impulse test are used. These tests are used complementarily to each other for diagnosing VN, and can also help to exclude the possibility of central vertigo [11-13]. Although formal diagnostic criteria have not been available, unilateral caloric paresis has been the diagnostic hallmark of VN [1,3]. In this study, some patients showed definite clinical characteristics of $\mathrm{VN}$, but they revealed minimal asymmetry on the caloric test and their values of $\mathrm{CP}$ were within the normal limit.

Bithermal caloric testing is a popular and classical means of evaluating peripheral vestibular function. Caloric irrigation assesses the VOR for mainly horizontal canal function by using temperature stimulation in the semicircular canals, which induces movement of the endolymph [14]. Caloric testing allows not only differentiation between peripheral and central lesions but also assessment of the severity of the disease. A caloric test shows hypo- or unresponsiveness for an affected horizontal canal in $\mathrm{VN}$, and this abnormal finding is usually maintained for more than a year [15]. With this evidence, unilateral $\mathrm{CP}$ has been considered to be a meaningful diagnostic marker of VN. However, the protocol for caloric testing (bithermal or monothermal) and the standard value of CP ( $20 \%-25 \%$ of CP) differs between laboratories, and there is no standardized qualified references.

There are very few studies of normal or MCP in patients with VN. Halmagyi et al. [16] presented cases of acute vertigo with a normal caloric response, but with pathological VEMP, with sparing of the superior vestibular nerve as inferiorVN. Another study reported that the subsequent conversion to unilateral CP seen with progression from isolated inferior $\mathrm{VN}$ to labyrinthitis [17]. However, in the present study, we excluded patients with isolated inferior vestibular nerve involvement and included those patients who showed clinical findings consistent with involvement of the superior vestibular nerve. Our data therefore cannot be explained by selective sparing of horizontal canal function.

Other studies have suggested that inconsistent caloric responses are influenced by various factors, such as visual fixation, the alertness of the patient, the time of the testing, heat conductivity of the ear, and test repetition [18], as well as by the degree of central compensation and previous vestibular disease [19]. In this study, we had totally excluded a history of vestibular disease, controlled the time of testing, and the same test method was applied by the same experienced examiner in all cases. We found that the $\mathrm{CP}$ value was consistent with the results of other vestibular function tests as well as with the clinical features.

During the acute phase of VN, most patients feel severely ill for about 1-3 days. Thereafter, spontaneous nystagmus becomes suppressed by fixation in the primary position [1]. During the recovery of peripheral vestibular function, spontaneous nystagmus transiently reverses direction, this is known to be related to central compensation, and reflects a tonic imbalance secondary to compensation [1]. In our study, transient direction change of spontaneous nystagmus was seen more frequently in the MCP group. This finding suggests that faster recovery with central compensation can be expected in the minimal paresis group. Analyzing the direction of DP, CP group patients showed contralateral DP dominantly but in the MCP group, the direction of DP was more dispersed by ipsilateral and contralateral side. This result also showed consistency for our analysis of spontaneous nystagmus because DP reflects dynamic status of spontaneous nystagmus at the point of caloric testing.

In conclusion, Patients with normal caloric responses ( $<25 \%)$ have a similar epidemiological background and clinical manifestations as conventional ( $\mathrm{CP} \geq 25 \%) \mathrm{VN}$ patients, but their symptoms resolve more rapidly and they have a higher incidence of recovery nystagmus. Furthermore, the MCP group of patients have a higher gain, especially for lower frequencies in the SHA test, and higher SOT scores than the CP group of patients. These could support that MCP group is considered as a milder type of VN. Not only the existence of canal paresis, but also the value of canal paresis, could help to predict the clinical course, and also could be a prognostic factor for the $\mathrm{VN}$ patients.

\section{CONFLICT OF INTEREST}

No potential conflict of interest relevant to this article was reported.

\section{ACKNOWLEDGMENTS}

This study was supported by the Inha University Research Grant, Incheon, Korea.

\section{REFERENCES}

1. Strupp M, Brandt T. Vestibular neuritis. Semin Neurol. 2009 Nov; 29(5):509-19.

2. Goddard JC, Fayad JN. Vestibular neuritis. Otolaryngol Clin North Am. 2011 Apr;44(2):361-5.

3. Jeong SH, Kim HJ, Kim JS. Vestibular neuritis. Semin Neurol. 2013 Jul;33(3):185-94.

4. Coats AC. Vestibular neuronitis. Trans Am Acad Ophthalmol Otolaryngol. 1969 May-Jun;73(3):395-408.

5. Anttinen A, Lang AH, Aantaa E, Marttila R. Vestibular neuronitis; a 
neurological and neurophysiological evaluation. Acta Neurol Scand. 1983 Feb;67(2):90-6.

6. Schuknecht HF, Kitamura K. Second Louis H. Clerf Lecture. Vestibular neuritis. Ann Otol Rhinol Laryngol Suppl. 1981 Jan-Feb;90(1 Pt 2):1-19.

7. Cooper CW. Vestibular neuronitis: a review of a common cause of vertigo in general practice. Br J Gen Pract. 1993 Apr;43(369):164-7.

8. Jongkees LB, Maas JP, Philipszoon AJ. Clinical nystagmography: a detailed study of electro-nystagmography in 341 patients with vertigo. Pract Otorhinolaryngol (Basel). 1962;24:65-93.

9. Arbusow V, Schulz P, Strupp M, Dieterich M, von Reinhardstoettner A, Rauch E, et al. Distribution of herpes simplex virus type 1 in human geniculate and vestibular ganglia: implications for vestibular neuritis. Ann Neurol. 1999 Sep;46(3):416-9.

10. Theil D, Arbusow V, Derfuss T, Strupp M, Pfeiffer M, Mascolo A, et al. Prevalence of HSV-1 LAT in human trigeminal, geniculate, and vestibular ganglia and its implication for cranial nerve syndromes. Brain Pathol. 2001 Oct;11(4):408-13.

11. Cnyrim CD, Newman-Toker D, Karch C, Brandt T, Strupp M. Bedside differentiation of vestibular neuritis from central "vestibular pseudoneuritis”. J Neurol Neurosurg Psychiatry. 2008 Apr;79(4): $458-60$.
12. Mandala M, Nuti D, Broman AT, Zee DS. Effectiveness of careful bedside examination in assessment, diagnosis, and prognosis of vestibular neuritis. Arch Otolaryngol Head Neck Surg. 2008 Feb;134 (2):164-9.

13. Newman-Toker DE, Kattah JC,Alvernia JE, Wang DZ. Normal head impulse test differentiates acute cerebellar strokes from vestibular neuritis. Neurology. 2008 Jun;70(24 Pt 2):2378-85.

14. Baloh RW, Honrubia V, Kerber KA. Baloh and Honrubia's clinical neurophysiology of the vestibular system. 4th ed. New York (NY): Oxford University Press; 2011.

15. Choi KD, Oh SY, Kim HJ, Koo JW, Cho BM, Kim JS. Recovery of vestibular imbalances after vestibular neuritis. Laryngoscope. 2007 Jul;117(7):1307-12.

16. Halmagyi GM, Aw ST, Karlberg M, Curthoys IS, Todd MJ. Inferior vestibular neuritis. Ann NYAcad Sci. 2002 Apr;956:306-13.

17. Kim JS, Kim HJ. Inferior vestibular neuritis. J Neurol. 2012 Aug;259 (8):1553-60.

18. Proctor L, Glackin R. Factors contributing to variability of caloric test scores. Acta Otolaryngol. 1985 Sep-Oct;100(3-4):161-71.

19. Baertschi AJ, Johnson RN, Hanna GR.A theoretical and experimental determination of vestibular dynamics in caloric stimulation. Biol Cybern. 1975 Nov;20(3-4):175-86. 